\title{
Efficient CRISPR/Cas9 genome editing in a salmonid fish cell line using a lentivirus delivery system
}

\author{
Remi L. Gratacap ${ }^{1 *}$ (D), Tim Regan ${ }^{1}$, Carola E. Dehler ${ }^{2}$, Samuel A. M. Martin², Pierre Boudinot ${ }^{3}$, Bertrand Collet $^{3}$ and \\ Ross D. Houston ${ }^{1 *}$
}

\begin{abstract}
Background: Genome editing is transforming bioscience research, but its application to non-model organisms, such as farmed animal species, requires optimisation. Salmonids are the most important aquaculture species by value, and improving genetic resistance to infectious disease is a major goal. However, use of genome editing to evaluate putative disease resistance genes in cell lines, and the use of genome-wide CRISPR screens is currently limited by a lack of available tools and techniques.

Results: In the current study, we developed an optimised protocol using lentivirus transduction for efficient integration of constructs into the genome of a Chinook salmon (Oncorhynchus tshwaytcha) cell line (CHSE-214). As proof-of-principle, two target genes were edited with high efficiency in an EGFP-Cas9 stable CHSE cell line; specifically, the exogenous, integrated EGFP and the endogenous RIG-I locus. Finally, the effective use of antibiotic selection to enrich the successfully edited targeted population was demonstrated.

Conclusions: The optimised lentiviral-mediated CRISPR method reported here increases possibilities for efficient genome editing in salmonid cells, in particular for future applications of genome-wide CRISPR screens for disease resistance.
\end{abstract}

Keywords: CRISPR, Lentivirus, Gene editing, CHSE, Salmon, Disease resistance

\section{Background}

Aquaculture is the fastest growing food production sector, and has overtaken capture fisheries as the primary source of seafood for human consumption [1]. However, farmed production of finfish, shellfish, and crustacean species all suffer from infectious diseases that can have negative impacts on animal welfare, the environment, and on commercial viability, constraining future expansion. Selective breeding for improved disease resistance is a promising avenue to tackle these diseases, and has

\footnotetext{
* Correspondence: Remi.Gratacap@ed.ac.uk; Ross.Houston@roslin.ed.ac.uk ${ }^{1}$ The Roslin Institute, University of Edinburgh, Easter Bush campus, Midlothian, UK

Full list of author information is available at the end of the article
}

been widely practiced for Atlantic salmon (Salmo salar) and other salmonid species [2, 3]. Family-based selection based on siblings trait recording is now typically augmented with the use of molecular genetic markers, either via marker-assisted selection (based on markers linked to quantitative trait loci, QTL) or genomic selection using genome-wide markers to predict breeding values [4]. However, to date, little is yet known about the functional genes and variants underlying this genetic resistance to disease.

Genome editing using reprogrammed CRISPR/Cas9 systems has emerged as a revolutionary tool to make specific and targeted changes to genomes of species' of interest. CRISPR/Cas9 can facilitate identification and 
characterisation of specific functional variants underlying QTL affecting the trait of interest. The technology facilitates precise gene knockout, activation or inhibition, and can also allow targeted deletion, insertion and even epigenetic modification of genomic DNA [5]. As such, CRISPR/Cas9 can be applied to test targeted perturbation of candidate genes and variants within QTL regions, to assess the consequence on the trait of interest. This knowledge raises the possibility of enhancing genomic selection accuracy via increased weighting on functional variants. In addition, genome editing can potentially by applied to create de novo variation, or to introduce favourable alleles segregating in closely related strains or species [6].

The aforementioned genome editing approaches typically focus on a single target locus, and there are several examples of successful CRISPR editing of single loci in vivo in farmed fish species (e.g. [7, 8]), reviewed in [6]). CRISPR/Cas9 has also been successfully applied in salmonid cell culture models to investigate specific components of the interferon pathway [9]. Another exciting application that has emerged in recent years is the development of genome-wide CRISPR knock out screens in cell culture models [10]. This involves creating a library of tens/hundreds of thousands of guide RNAs (gRNA) targeting either every gene in the genome of the species of interest, or targeting non-translated regions such as enhancers or miRNA [11]. These guides are then synthesised, packaged into a lentivirus vector, and transduced into a cell line constitutively expressing Cas9 (or alternatively the transduced construct can also code for the Cas9 protein). The lentivirus dose used results in approximately one gRNA integration per cell. The cell pool is then screened (e.g. using a pathogen challenge) and the selected cells (surviving, fluorescently labelled, or another marker of selection) sequenced. The enrichment or depletion of gRNAs compared to the control population informs on the role of their target genes in the phenotype under investigation. This approach has led to fundamental host-pathogen discoveries, particularly in virology as the cell intrinsic nature of the innate immune response is very well suited to interrogation with this platform. Such screens have led to the discovery of the Norovirus receptor [12] and the role of endoplasmic reticulum membrane complex in Zika virus infection [13]. Genome-wide screens have been applied in several species, from humans to fly [14], including parasites such as Toxoplasma gondii [15], and plants [16] but has yet to be applied in farmed fish species, where it could have major potential for discovering genes involved in disease resistance, and improving knowledge of hostpathogen interaction.

There are currently several barriers and knowledge gaps preventing the application of genome-wide screens in fish. However, one major barrier was overcome when a potentially suitable cell line was created by Dehler et al. [17], using a random plasmid integration event to stably express Cas9 in the Chinook salmon (Oncorhynchus tshawytscha) cell line (CHSE-EC), a cell line frequently used to study viral diseases of interest to farmed salmonid production. Further, this transgenic line was also modified to stably express EGFP, and Dehler et al. used electroporation of gRNA to successfully knockout the integrated EGFP locus, reporting approximately $35 \%$ successful editing. Using this approach, they developed clonal lines of CHSE STAT2 KO and explored the role of this gene in antiviral immunity [9]. Alternatively, the genome editing cargo can be delivered by transient transfection of a plasmid expressing Cas9 and gRNA. Escobar et al., [18] successfully edited the genome in the CHSE cell line but reported a low transfection/expression efficiency $(10 \%)$ and did not report editing efficiency. To harness the CHSE-EC (or similar Cas9 stable) cell lines as a platform for high-throughput screens, an efficient lentivirus delivery system for the genome-wide library would be highly desirable.

Lentiviral transduction of Cas9 and gRNA has several advantages over other methods, such as electroporation of ribonucleoprotein or transient plasmid transfection. It efficiently integrates into the genome, enabling the creation of stable cell lines, and allows for the integration of antibiotic resistance markers and fluorescent reporters to perform enrichment of edited cells. A multitude of lentivirus plasmid constructs already exist, and have been successfully tested in various species. For these reasons, lentivirus is the delivery method of choice for genome-wide libraries, but has not yet been developed in fish cell lines. In the current study, an efficient lentivirus-based method for genome editing in a salmonid fish cell line is presented. The delivery of lentivirus was optimised to allow integration of a transgene at high efficiency. Using a lentivirus delivered gRNA in the salmonid cell line CHSE-EC, a very high efficiency of genome editing was obtained, after antibiotic enrichment of cells containing both Cas9 and specific gRNA. Finally, as proof of principle, the method was applied to establish a polyclonal cell line enriched for retinoic acidinducible gene-I-like (RIG-I) knock-out cells.

\section{Results}

\section{Efficient transduction of Chinook salmon cells with lentivirus}

To improve CRISPR/Cas9 delivery and genome editing efficiency, lentiviral transduction was optimised for use in salmonid cell lines. This was initially performed using a fluorescent reporter construct (CMV:EGFP) in the Chinook salmon cell line (CHSE-214, referred to hereafter as CHSE). To optimise the transduction efficiency, 
three major variables were tested $[19,20]$; specifically (i) the impact of incubation temperature; (ii) the impact of including a spinfection (or spinoculation); and (iii) the impact of the duration of the incubation. Flow cytometry was used to determine the efficiency of transduction by measuring the number of fluorescent cells (therefore assumed to be transduced with the CMV-EGFP), with the outputs from the optimised settings shown in Fig. 1a and $b$.

Temperature of incubation had a major impact on transduction efficiency, with an increased transduction efficiency from 1 to $63 \%$ as the temperature was increased from $17^{\circ} \mathrm{C}$ to $22^{\circ} \mathrm{C}$ (Fig. 1c). Further increase in temperature to $28^{\circ} \mathrm{C}$ for $4 \mathrm{~h}$ (heat shock) followed by $22^{\circ} \mathrm{C}$ incubation (denoted 28-22) resulted in a minor increase in transduction efficiency, from 63 to $73 \%$ (Fig. 1c), but overnight incubation at $28{ }^{\circ} \mathrm{C}$ caused mortality in the CHSE cells (data not shown). Using the settings of $22^{\circ} \mathrm{C}$ incubation for $24 \mathrm{~h}$ and neat supernatant of lentivirus, we included a spinfection step and show an improvement of transduction efficiency from 47 to $63 \%$ (Fig. 1c). Finally, reducing the incubation time of the cells with the lentivirus from $24 \mathrm{~h}$ to $4 \mathrm{~h}$ reduced the transduction efficiency from 63 to $43 \%$ for $22{ }^{\circ} \mathrm{C}$ incubation and 73 to $53 \%$ for $28-22$ incubation group (Fig. 1d). Therefore, we propose an optimised protocol for efficient transduction of CHSE cells using neat lentivirus supernatant on suspended cells, together with a spinfection step ( $2 \mathrm{~h}$ at $1000 \mathrm{x} \mathrm{g})$ and incubation for $24 \mathrm{~h}$ at $22^{\circ} \mathrm{C}$ (Fig. 1a, b). These optimised settings were used for downstream experiments.

\section{Efficient editing of the Chinook salmon genome using a lentivirus system}

After optimising the transduction conditions by integrating a GFP expressing construct into the CHSE cell line, a modified CHSE cell line stably expressing Cas9 and EGFP (CHSE-EC [17]) was used to test Cas9-mediated genome editing in these cells. A plasmid, containing a human U6 promoter to drive the expression of a second-generation gRNA scaffold and the puromycin antibiotic resistance gene was used [21]. The use of the human U6 promoter to drive the expression of a gRNA
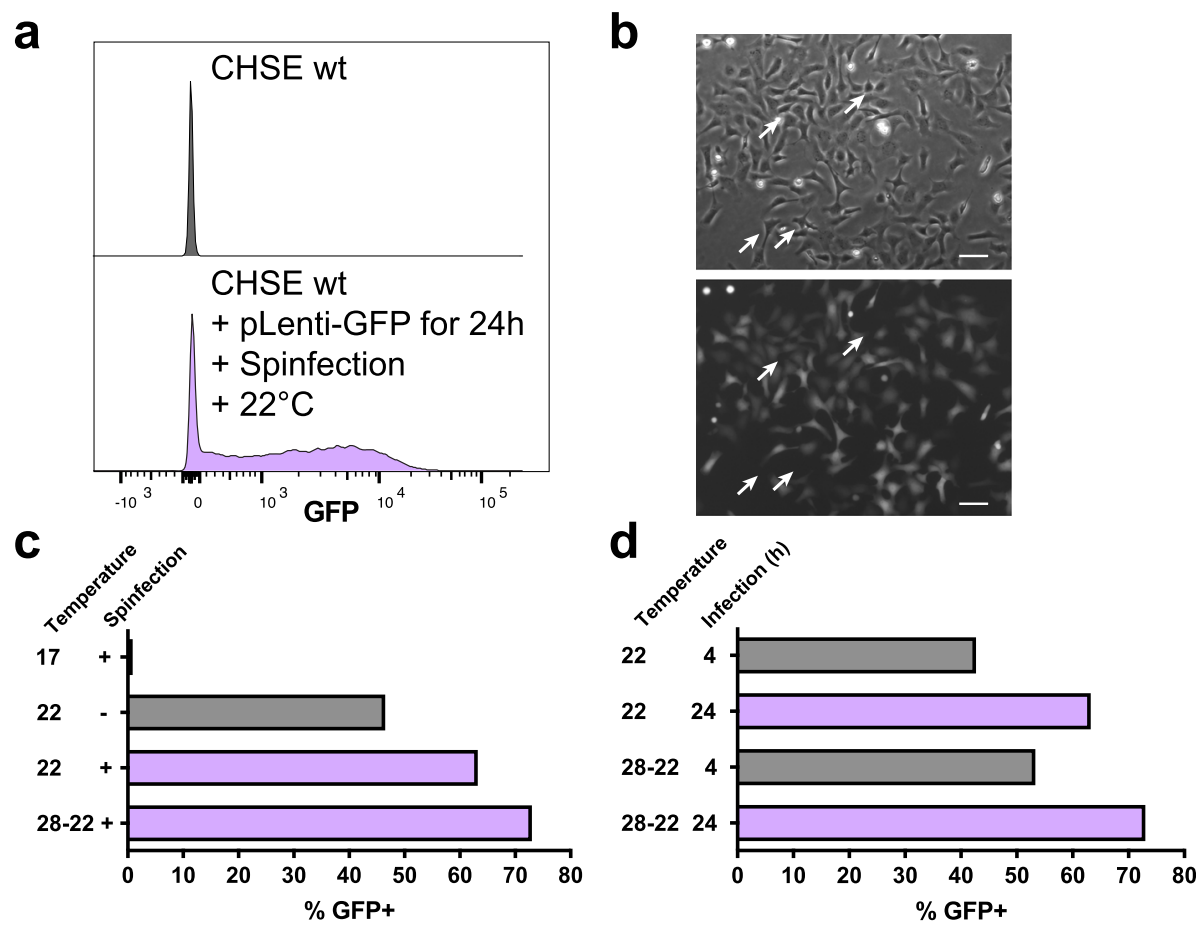

Fig. 1 CHSE salmon cells are efficiently transduced with lentivirus. a Efficient transduction of CMV:EGFP in CHSE Chinook salmon cell line by lentivirus. Salmon cells were spinfected at $22^{\circ} \mathrm{C}$ with neat lentivirus supernatant for $2 \mathrm{~h}$ at $1000 \mathrm{xg}$. The cells were incubated for $24 \mathrm{~h}$ and the media was replaced. After 2 weeks of expansions, fluorescence was recorded by flow cytometry using CHSE wt (not transduced) as control. Split histogram of control cells (top) and pLenti-GFP transduced CHSE with optimal conditions (bottom). Data were normalised to Mode (relative percentage of cells rather than number). b Representative image of CHSE cells 8 days post transduction with pLenti-GFP. Differential Interference Contrast (top) and GFP (bottom) channels are represented. Arrows point to GFP negative cells. Scale bar 20 um. c-d Different conditions of the optimisation protocol with optimal conditions in purple. $\mathbf{c}$, Incubation temperature and spinfection influences the efficiency of transduction. Cells were transduced with neat lentivirus supernatant, spinfected for $2 \mathrm{~h}$ at $1000 \times \mathrm{g}$ (or not) and incubated at $17^{\circ} \mathrm{C}(17), 22^{\circ} \mathrm{C}(22)$ or heat shocked for $4 \mathrm{~h}$ at $28^{\circ} \mathrm{C}$ followed by $22^{\circ} \mathrm{C}$ for 2 weeks (28-22) until flow cytometry. $\mathbf{d}$, Incubation of the cells with the virus for $4 \mathrm{~h}$ reduces the efficiency of transduction. CHSE were transduced with neat lentivirus dose at different temperatures and media was changed after 4 or $24 \mathrm{~h}$ 
has previously been reported to be inefficient in this salmonid cell line [18]. Therefore, to first test this, the gRNA sequence for EGFP [22] was cloned in this plasmid (pKLV2_EGFP), and transduced CHSE-EC cells using the optimised protocol described previously. Editing efficiency was determined by loss of fluorescence for EGFP editing as well as sequencing of pooled populations of cells (EGFP and RIG-I targets) followed by deconvolution of the pooled sequencing chromatogram using the ICE online software (Synthego Inc., example in Additional file 2: FigS2).

Neat lentivirus supernatant was used to transduce CHSE-EC, which resulted in up to $89.9 \%$ reduction in fluorescent cells by flow cytometry (Fig. 2a, b). A 10-fold dilution of the lentivirus supernatant was also used to validate the antibiotics enrichment. This yielded a $42.9 \%$ loss of fluorescence, which could be effectively enriched using puromycin selection for 1 week (Additional file 1: Fig. S1), to $82.5 \%$ loss (Lo + Puro, Fig. 2b, c). In close agreement with the flow cytometry data, Sanger sequencing analysis of the target region showed $70 \%$ editing (cutting efficiency using ICE, Synthego Inc) of the cells in the neat supernatant group (and 58\% in the low dose enriched, Fig. 2c). Deconvolution of the chromatogram edit patterns (ICE analysis) shows that the majority of the edited sequences had a $1 \mathrm{bp}$ insertion (27\%) and only $15 \%$ of the sequences were estimated to be unedited (Fig. 2d).

In summary, the results show that the pKLV2 plasmid (containing the human U6 promoter) is functional and effective at transcribing gRNA in the CHSE cell line, and that the lentivirus delivery strategy (either neat, or diluted together with antibiotic selection for enrichment) leads to very high genome editing efficiency.

To validate that the editing strategy was efficient on a salmon gene, the Chinook salmon retinoic acidinducible gene-I-like (RIG-I) locus (DDX58, NCBI Gene LOC112222314) was targeted, by creating a pKLV2 RIG-I construct. This gene was chosen as it is central in the antiviral defense against RNA virus [23], it exists as a single copy in the chinook salmon genome, and because knockout cell lines may be useful models for future studies of host response to these viruses. The CHSE-EC cells were transduced with pKLV2_RIG-I supernatant, followed by puromycin selection for 7 days. At least $47 \%$ of the cells were edited at the desired locus (Puro-, Fig. $2 \mathrm{e}$ ) and this was enriched to $60 \%$ using puromycin selection (Puro+, Fig. 2e). Similarly to the EGFP edited cells (Fig. 2d), 1 bp insertion was the most common type of edit detected (Fig. 2f). We also sequenced the 2 top offtargets regions predicted by CRISPOR (TableS1) and evaluated editing using Sanger sequencing as previously described (FigS2). No editing was detected in either of the regions (FigS3). These results demonstrate that endogenous genes in the chinook salmon CHSE cell line can be targeted for genome editing using lentivirus.

Taken together, these results show that the lentivirus delivery strategy described herein, together with the Cas9 expressing CHSE cell line (CHSE-EC) can easily, rapidly and efficiently be used to edit the genome of salmonid cells, and suggests that the method could be applied to the new generation of CRISPR/Cas9 platforms such as base editors and CRISPR activation/inhibition.

\section{Discussion}

The results of the current study show efficient lentivirus transduction in the salmonid cell line CHSE-214 and the first demonstration of lentivirus-mediated editing in salmonid fish cells. The optimised protocol described results in the most efficient genome editing of a salmonid cell line to date. However, it is important to note that the results are based on single experimental replicates, and it will be beneficial to repeat the editing in this cell line, as well as to test in other fish cell lines to demonstrate the versatility of the method. Nonetheless, this study paves the way to high-throughput genome editing in salmonid cells, and for simpler generation of transgenic cell lines.

Retroviruses have widely been used to infect animal cells and to deliver cargo which can be integrated or not in the genome [24]. Over 25 years ago, it was shown that fish cells (zebrafish, Danio rerio ZF4, Rainbow trout, Oncorhynchus mykiss RTG-2 and Chinook salmon, CHSE-214) could be transduced with retrovirus (Moloney murine leukemia virus, MLV) after subtyping it with the VSV-G envelope glycoprotein (from Vesicular Stomatitis Virus) [25]. Thereafter, several viral delivery vectors have been tested for the expression of genes (integrative or non-integrative system) in several fish cells and in vivo, including MLV [26], Semliki Forest Virus (SFV) [20], lentivirus, adeno-virus (Ad) and adeno-associated virus (AAV) [27-29], with levels of efficiency varying from very high [Ad in zebrafish cells [27]] to low [MLV in medaka stem cells [26]]. Therefore, the transduction of cell lines has been shown to be efficient with the retrovirus system, but its adoption to edit the genome of fish cell lines has not been demonstrated.

In the present study, the delivery method of the retrovirus-derived second-generation lentivirus system [30] was optimised to stably integrate an EGFP construct in the salmonid cell line CHSE-214. Transduction efficiency is dependent on temperature and is almost completely blocked at incubation below $17^{\circ} \mathrm{C}$. The cell type and temperature influence on lentivirus transduction efficiency has been reported previously in mammalian cells $[19,31]$. In fish cell lines, SFV 
a

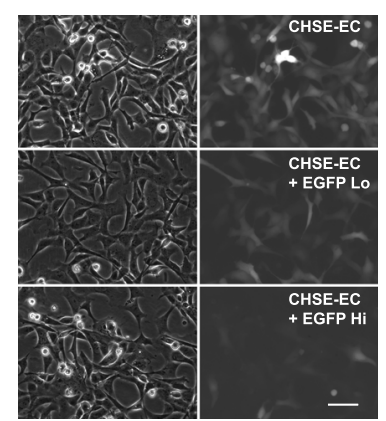

C

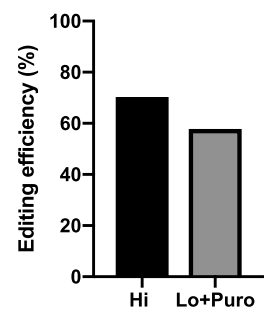

e

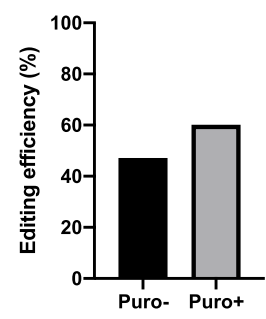

b

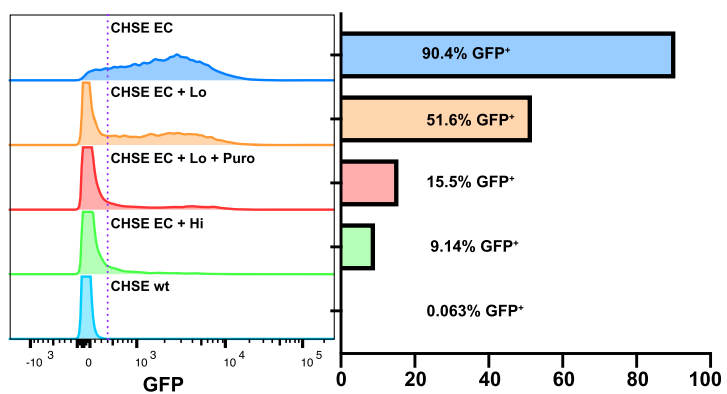

d

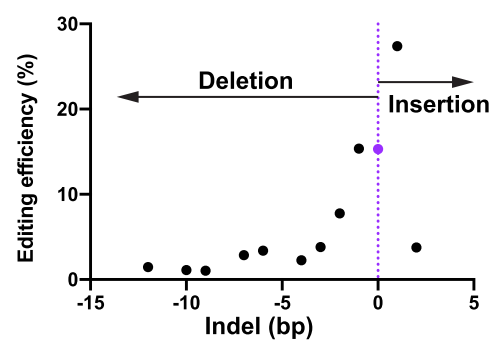

f

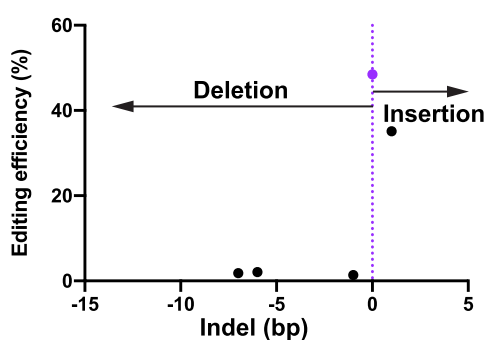

Fig. 2 The genome of CHSE-EC salmon cells is efficiently edited with lentivirus. a-d Efficient editing of EGFP in CHSE-EC Chinook salmon cell line by lentivirus. CHSE-EC cells were spinfected for $2 \mathrm{~h}$ at $1000 \mathrm{~g}$ with neat $(\mathrm{Hi})$ and 1:10 dilution (Lo) of lentivirus supernatant and incubated at $22^{\circ} \mathrm{C}$. After 2 weeks of expansion, puromycin was added to the Lo group $(0.25 \mu \mathrm{g} / \mathrm{mL}$, for 1 week, Lo + Puro). Fluorescence was imaged by epifluorescence microscopy (a) and recorded flow cytometry (b) using CHSE wt and CHSE-EC (not transduced) as controls. Split histogram of fluorescence and corresponding proportion histogram of control cells (top and bottom) and CHES-EC transduced with high and low concentration (with and without puromycin treatment) lentivirus supernatant. Scale bar in a represents $20 \mu \mathrm{m}$. c Genome editing efficiency in CHSE-EC by Sanger sequencing of PCR amplified product of the target loci, estimated by ICE analysis. $\mathbf{d}$ Detail of indels frequency in EGFP edited $\mathrm{Hi}$ group (from panel c), estimated by ICE analysis. Purple dot denotes the unedited sequence (0 bp). e-f Efficient editing of RIG-I in CHSE-EC. e Genome editing of the RIG-I gene in CHSE-EC. CHSE-EC cells were transduced similar to EGFP targeting (Puro-) and selected with puromycin (Puro+) and efficiency estimated by ICE analysis of Sanger sequencing chromatogram from the PCR amplified target region. $\mathbf{f}$ Detail of indels frequency in RIG-I edited Puro- group (from panel e), estimated by ICE analysis. Purple dot denotes the unedited sequence (0 bp)

infection was reduced 1000 -fold in the Atlantic salmon F95/9 cell line and completely blocked in CHSE214 as the temperature was decreased from 25 to $15{ }^{\circ} \mathrm{C}[20]$. This temperature restriction is fortunately not a limiting issue as most fish cell lines, derived from cold or warm water species, are adapted to temperatures of $20^{\circ} \mathrm{C}$ or above [32]. Similar to several studies in mammalian cells, adding a spinfection step $(1000 \mathrm{x} \mathrm{g}$ for 1-2 h) greatly enhanced the transduction efficiency of LV in the current study $[19,33]$. The relative ease of stably integrating a reporter construct and possibly tagging the native protein [34] is likely to help with in vitro research in salmonid cell lines.
A major advantage of lentivirus transduction is the possibility to integrate an antibiotic resistance cassette to enrich the transduced cells. Using this approach, it is possible to use a relatively low viral dose, which ensures that a single copy of the insert is integrated in the genome, and subsequently enrich the transduced cells with antibiotic treatment to very high proportion of cells. The high transduction efficiency and genome editing achieved offer advantages over less efficient gene editing methods such as the possibility to directly use the cells generated as a pooled population without the tenuous process of single cell cloning. However, it should be noted that lentivirus, like most integrating retroviruses, 
tend to integrate at the site of active transcription (active genes) and that an immune response (interferon stimulated genes) has been reported with lentivirus transduction (i.e. infection) $[35,36]$.

By using the CHSE-EC cell line which constitutively expressed Cas9, in combination with a lentivirus packaging a gRNA construct targeting EGFP or RIG-I, the genome of the salmonid cells was edited at very high efficiency. Using this strategy, the previously reported editing efficiency of $35 \%$ by electroporation of the gRNA [17] was improved to almost 90\% editing of EGFP (as assessed by flow cytometry). Nonetheless, it should be noted that electroporation of difficult-to-transduce cells has been extremely successful and is also one of the main approaches to edit the genome of mammalian immune cells [37].

The expected positive correlation was observed between the editing efficiency estimated by Sanger sequencing and the phenotype observed when targeting the exogenous EGFP gene in the CHSE-EC salmonid cell line. However, disrupting specific pathways by precise targeting of endogenous genes might be more challenging, due to the recent salmonid whole genome duplication (approx. 95 million years ago, [38]). As such, it will be necessary in many cases to target all paralogs to achieve the desired phenotype. Moreover, several mechanisms have been hypothesized to explain genetic robustness (i.e. a mutation not exhibiting the expected phenotype), such as functional redundancy [39], rewiring of genetic networks [40], adaptive mutations [41] or transcriptional adaptation [42]. Finally, off-target are an ever-present concern when editing the genome with Cas9 and great care has to be used then choosing gRNA sequences to minimise the possibilities of off-target. In the present study, the 2 top off-target regions (Table S1) were sequenced and showed no editing (FigS3).

\section{Conclusions}

The optimised protocol described in this study allows for relatively fast and cost-effective gene editing in Chinook salmon cells to generate reporter or CRISPR KO lines. Cell lines from other salmonid species such as Atlantic salmon might be readily transduced with similar lentivirus systems. However, salmonid cell lines are generally slow growing and difficult to transfect with plasmids, with the CHSE being the most amenable to clonal expansion. Therefore, development of embryonic cell lines from other salmonid species may be a useful approach to generate appropriate cell lines for CRISPR KO experiments in other commercially relevant aquaculture species.

The prospect of developing and applying a genome wide CRISPR $\mathrm{KO}$ screen in fish cell lines is very attractive for many reasons $[6,10]$ and the present system paves the way towards such a platform for salmonids. This has the potential to be transformative for the testing of candidate disease resistance genes generated by Genome-Wide Association Studies as well as de novo discovery of genes involved in host-pathogen interactions in fish.

\section{Methods \\ Cell culture}

CHSE-214 cell line from Chinook salmon (ECACC \#91041114) was obtained from Sigma (catalogue \#00021714). CHSE-EC were a kind gift from Bertrand Collet and previously described in [17]. Both salmonid cell lines were cultured in L15 media (Sigma L1518) supplemented with $10 \%$ heat inactivated fetal bovine serum (FBS, Gibco) and 1x Pen/Strep (P/S) antibiotics (Gibco). Cells were cultured at $22^{\circ} \mathrm{C}$ (or see optimisation for other temperatures) without $\mathrm{CO}_{2}$ and split 1:4 at $80 \%$ confluency. Lenti-X $293 \mathrm{~T}$ (Takara/Clontech, catalogue \#632180) were cultured in DMEM + 10\% FBS, Penicillin, Streptomycin and Glutamine, Sodium Pyruvate and NEAA (all from Gibco). Cells were cultured at $37{ }^{\circ} \mathrm{C}$ with $5 \% \mathrm{CO}_{2}$ and split $1: 20$ at $80 \%$ confluency. Puromycin (Cayman chemicals) was used for antibiotic selection at $0.25 \mu \mathrm{g} / \mathrm{mL}$ for 7 days (Additional file 1: FigS1). The media was changed every $3-4$ days.

\section{gRNA design}

All gRNAs were designed using CRISPOR (www.crispor. tefor.net) and the Chinook salmon reference genome (NCBI GCF_002872995.1, Otsh_v1.0) was used for offtarget evaluation. The top 2 off-target sites were selected from the aggregate of MIT and CDF off-target scores (Supplementary TableS1). The oligos and PCR primers spanning the edited locus (400-600 bp), synthesised by IDT or Sigma can be found in Table 1. All oligos (Table 1) were resuspended to $100 \mu \mathrm{M}$ in $1 \mathrm{x}$ TE.

\section{Plasmids constructs (Table 2)}

pKLV2-U6gRNA5(BbsI)-PGKpuro2ABFP-W (pKLV2) was a gift from Kosuke Yusa (Addgene plasmid \# 67974). Specific gRNA for EGFP and RIG-I (Table 2, underlined) were cloned according to Golden Gate reaction from ZhangLab protocol (Addgene SAM library sgRNA cloning protocol) using BbsI-HF (NEB) and Stbl3 competent E. coli cells (Thermo Fisher) for transformation. Briefly, $1 \mu \mathrm{L}$ of each oligo $(100 \mu \mathrm{M})$ were mixed with $1 \mu \mathrm{L}$ of $\mathrm{T} 4$ ligation buffer (NEB) and $7 \mu \mathrm{L}$ of water. The mix was heated to $95^{\circ} \mathrm{C}$ for $5 \mathrm{~min}$ and cooled to room temperature. $1 \mu \mathrm{L}$ of the annealed oligos was mixed with $25 \mathrm{ng}$ of pKLV2, $1 \mu \mathrm{L}$ of T4 ligation buffer, $9 \mu \mathrm{L}$ of water and $0.5 \mu \mathrm{L}$ of BbsI enzyme and $0.5 \mu \mathrm{L}$ of T4 ligase enzyme (200 U, NEB). The mix was incubated for 10 cycles of $5 \mathrm{~min}$ at $37^{\circ} \mathrm{C}$ and $5 \mathrm{~min}$ at 
Table 1 Primers used in the study (from IDT or Sigma). gRNA target sequence underlined

\begin{tabular}{lll}
\hline Primers name & Primer sequence & Annealing temp $\left({ }^{\circ} \mathrm{C}\right)$ \\
\hline EGFP_Fw_gRNA & caccgggcgaggagctgttcaccg & $\mathrm{N} / \mathrm{A}$ \\
EGFP_Rv_gRNA & aaaccggtgaacagctcctcgccc & $\mathrm{N} / \mathrm{A}$ \\
EGFP_Fw_seq (CMV-F) & cgcaaatgggcggtaggcgtg & 63.5 \\
EGFP_Rv_seq & gctgaagcactgcacgccgt & $\mathrm{N} / \mathrm{A}$ \\
U6_Fw_Seq (hU6-F) & gagggcctatttcccatgatt & $\mathrm{N} / \mathrm{A}$ \\
Otsha_RIG-I_Fw_gRNA & caccgcgttccagaggttgcagag & $\mathrm{N} / \mathrm{A}$ \\
Otsha_RIG-I_Rv_gRNA & aaacctctgcaacctctggaacgc & 64 \\
Otsha_RIG-I_Fw_seq & aagtgtcaaagtacagcaattctagc & \\
Otsha_RIG-I_Rv_seq & cttccagggcatctagcagg & 64 \\
Otsha_RIG-I_OffT1_Fw_seq Offfasasfafgfaf'fl_Off1_Fw_sec & tgttgatctctgccttgcac & \\
Otsha_RIG-I_OffT1_Rv_seq & tatcccaccttggttgcttc & 64 \\
Otsha_RIG-I_OffT2_Fw_sec & cggacaacaggccattagat & \\
Otsha_RIG-I_OffT2_Rv_sec & tggcgtactgcactagcatc & \\
\hline
\end{tabular}

$23^{\circ} \mathrm{C}$. Five $\mu \mathrm{L}$ were transformed in $50 \mu \mathrm{L}$ competent $E$. coli (Stbl3, Thermo fisher). Four colonies were picked and grown in LB + Carbenicillin, the plasmids purified (NEB Monarch Plasmid miniprep) and sequenced to verify correct insertion using U6_Fw_seq primer (Table 1).

\section{Lentivirus production}

Supernatants containing lentiviral particles were produced by transient transfection of x293T cells using Lipofectamine 2000 (Invitrogen). To transfect one well of a 6 -well plate, $1.5 \times 10^{6} \times 293 \mathrm{~T}$ cells were plated in 4 $\mathrm{mL}$ of complete media and incubated overnight at $37^{\circ} \mathrm{C}$ (70\% confluency at $24 \mathrm{~h}$ ). The following day, $1.8 \mu \mathrm{g}$ of lentiviral vector, $1.8 \mu \mathrm{g}$ of psPAX2 (gift from Didier Trono, Addgene plasmid \# 12260) and $0.4 \mu \mathrm{g}$ of pMD2.G (gift from Didier Trono, Addgene \#12259) were added to $160 \mu \mathrm{L}$ of Opti-MEM (no phenol red, Gibco), $12.5 \mu \mathrm{L}$ of Lipofectamine 2000 was added to $140 \mu \mathrm{L}$ of Opti-MEM and both tubes incubated for 5 min at room temperature. The Lipofectamine 2000 mixture was then added to the plasmids mixture and further incubated for $20 \mathrm{~min}$ at room temperature. The transfection complex $(300 \mu \mathrm{L})$ was added dropwise to one well of x293T and incubated for $48 \mathrm{~h}$ at $37^{\circ} \mathrm{C}$ with $\mathrm{CO}_{2}$. Viral supernatant was then harvested, centrifuged at $500 \mathrm{x}$ g for $4 \mathrm{~min}$, filtered using a $0.45 \mu \mathrm{m}$ low protein retention syringe filter (Sartorius) and $0.5 \mathrm{~mL}$ aliquots were stored at $-80^{\circ} \mathrm{C}$.

\section{Lentiviral transduction}

For lentiviral transduction of CHSE or CHSE-EC, $50 \mu \mathrm{L}$ of cells $\left(4.10^{5}\right.$ cells $\left./ \mathrm{mL}\right)$ were mixed with $100 \mu \mathrm{L}$ of the lentiviral supernatant (at various dilutions) in a 96-well plate or $200 \mu \mathrm{L}$ of cells plus $400 \mu \mathrm{L}$ of viral supernatant in 24-well plate, and centrifuged for $2 \mathrm{~h}$ at $1000 \mathrm{x} \mathrm{g}$. Additionally, a lentivirus-free control condition (CHSEEC wt) was also included to validate the antibiotic selection procedure (i.e. 100\% death upon puromycin selection). Media was changed after $4 \mathrm{~h}$ or $24 \mathrm{~h}$. After transduction, cells were incubated at the indicated temperatures and split as needed. Cells were then expanded once reaching $80 \%$ confluence. Antibiotics treatment was carried out for 7 days and the surviving cells expanded once reaching $80 \%$ confluence. After 2 rounds of expansion, genomic DNA was isolated from a minimum of 50,000 cells (see 7.7 Sequencing).

\section{Flow cytometry}

Transduced cells were detached with trypsin, centrifuged and resuspended in PBS. The cells were kept on ice and flow cytometry was performed using a Fortessa-X20 (BD Biosciences). Events were gated to remove doublet cells and the GFP intensity of each cell was analysed with FlowJo 10.6.0 (Becton Dickinson). Transduction efficiency with CMV:EGFP construct (Fig. 1) was calculated by dividing the number of EGFP positive cells by total number of cells acquired (minimum 5000 cells).

Table 2 Plasmids used in this study (from Addgene, USA)

\begin{tabular}{lll}
\hline Plasmid construct & Plasmid name (Addgene) & Plasmid number (Addgene) \\
\hline pLenti CMV:EGFP_PGK:Puro & pLenti CMV GFP Puro (658-5) & 17,448, [43] \\
pLenti hU6:gRNA_PKG:Puro & pKLV2-U6gRNA5(Bbsl)-PGKpuro2ABFP-W & 67,974, [21] \\
\hline
\end{tabular}




\section{Sequencing}

Genomic DNA was extracted with QuickExtract buffer (Lucigen) by adding $30 \mu \mathrm{L}$ to a single well of a 96-well plate and incubating for $5 \mathrm{~min}$. The samples were then processed according to the manufacturer's instructions $\left(65^{\circ} \mathrm{C}\right.$ for $6 \mathrm{~min}$ and $98^{\circ} \mathrm{C}$ for $\left.2 \mathrm{~min}\right)$. PCR was performed with $50 \mu \mathrm{L}$ reactions using NEB Q5 and $1 \mu \mathrm{L}$ of the gDNA for 33 cycles amplification at optimal annealing temperature (see Table 1). Amplified products were purified using AMPure XP magnetic beads (Agencourt) according to the manufacturer's instructions. Purified PCR products were sequenced by GATC/Eurofins. Sequence (ab1 files) were analysed with Interference of CRISPR Edits software (www.ice.synthego.com, [44]) to determine the editing and knock-out (KO, frame-shift or early stop codon) efficiency, compared to controls (non-edited).

\section{Cell survival (puromycin resistance)}

CellTiter-Glo 2.0 (Invitrogen) was used for cell viability assays. Briefly, CHSE-EC cells were plated in 96well plate at 8000 cells per well and, after $24 \mathrm{~h}$, different concentrations of Puromycin (Cayman chemicals) were added to each well (in duplicates). After 7 days incubation at $22^{\circ} \mathrm{C}$, cells were rinsed in PBS and $120 \mu \mathrm{L}$ of diluted solution of CellTiter-Glo (1:10 in PBS) was added to each well. The plate was incubated in the dark for $30 \mathrm{~min}$ rocking at room temperature and $100 \mu \mathrm{L}$ of the solution was transferred to a flat bottom white wall 96-well plate (Greiner Bio-One). The luminescence was measured immediately using a Cytation 3 imaging reader and the Gen5 software (V3.03, both from BioTek).

\section{Supplementary information}

Supplementary information accompanies this paper at https://doi.org/10. 1186/s12896-020-00626-x.

\begin{abstract}
Additional file 1 Figure S1. Puromycin can be used to select for resistant cells. CHSE-EC cells were treated for 7 days with different concentrations of Puromycin and the survival was calculated by CellTiter-Glo. A concentration of $0.25 \mu \mathrm{g} / \mathrm{mL}$ of puromycin was found to be the minimal concentration to efficiently kill all non-antibiotic-resistant cells.
\end{abstract}

Additional file $\mathbf{2}$ Figure S2. Editing efficiency estimation, The analysis of the editing of pooled cell population samples using ICE online software. a. The chromatograms (.ab1 file) from the control (non-edited) and edited samples, along with the gRNA sequence are uploaded on ice. sythego.com. b. The platform verifies that the cut site corresponds to the start of the mixed population chromatogram and deconvolutes the picks to original sequences + or - a few bases. $c$. The results are presented as the percentage of each edited sample present in the pooled population contributing to the mixed chromatogram.

Additional file $\mathbf{3}$ Figure S3. Off-target editing. Off-target evaluation of RIG-I editing. a: The sequence of the top 2 off-target sites are represented along the gRNA sequence used for targeting RIG-I (red box in the middle). Blue letters indicate differences with the original sequence. An additional nucleotide was sequenced in the CHSE-EC cell line, not present in the published sequence (Otsh_v1.0, green box). b: Diagram representing the editing efficiency in the off-target regions (OffT1: ch7:7310172873,102,398 and OffT2: ch14:42341999-42,342,859). No off-target was detected by Sanger sequencing in either sample (Puro- and Puro+; all sequences, including CHSE-EC (wt) were compared to CHSEwt). c: Representative chromatogram from the sequencing of off-target region 1 (OffT1) in CHSEwt (Control sample, bottom track) and CHSE-EC-RIG-I Puro + (Edited Sample, top track).

Additional file 4 Table S1. Predicted Off-target sites. List of all predicted off-targets from CRISPOR website for 354 bp in RIG-I exon 2 (Tab1). The selected gRNA is labeled 224 forw and the list of off-targets can be found lines 2127-2240. Tab2 summarises the predicted off-targets for gRNA 224 forw. The sum of the two off-target scores (MIT and CDF) were calculated to rank the results. The 2 targets selected are highlighted.

\section{Abbreviations}

Ad: Adenovirus; AAV: Adeno-Associated Virus; Cas9: CRISPR associated protein 9; CMV: Cytomegalovirus; CRISPR: Clustered Regularly Interspaced Short Palindromic Repeats; EGFP: Enhanced Green Fluorescent Protein; gRNA: guide RNA; ICE: Inference of CRISPR Edits; KO: Knockout; MLV: Murine Leukemia Virus; LV: Lentivirus; PBS: Phosphate Buffer Saline; PCR: Polymerase Chain Reaction; PGK: Phosphoglycerate Kinase; Puro: Puromycin; QTL: Quantitative Trait Loci; RIG-I: Retinoic Acid Inducible Gene I; RNP: Ribonucleoprotein; SFV: Semliki Forest Virus; STAT2: Signal Transducer and Activator of Transcription 2; VSV: Vesicular Stomatitis Virus

\section{Acknowledgements}

We would like to acknowledge colleagues within Ross Houston's laboratory and Spring Tan for fruitful discussions as well as the Roslin Institute Bioimaging and Flow cytometry consortium.

\section{Authors' contributions}

$\mathrm{RH}, \mathrm{TR}$ and RG conceived and designed the study; RG performed the experiments; CD, SM, BC and PB contributed data and reagents; $\mathrm{RH}, \mathrm{TR}$ and $\mathrm{RG}$ wrote the paper. All authors read and contributed to the final manuscript.

\section{Authors' information}

N/A

\section{Funding}

The present study was funded by were funded by the Biotechnology and Biological Sciences Research Council (BB/R008612/1, BB/S004343/1 to RH and RG; grant BB/R008973/1 to SM and CD) and the Institute Strategic Programme Grants (BBS/E/D/20002172, BBS/E/D/30002275 and BBS/E/D/ 10002070, to $\mathrm{RH}$ and $\mathrm{RG}$ ). The funders had no roles in the study design, data collection and analysis, decision to publish or preparation of the manuscript.

\section{Availability of data and materials}

The datasets used and/or analysed during the current study (flow cytometry and Sanger sequencing files) are available from the corresponding authors on reasonable request.

Ethics approval and consent to participate

Not required for this study.

\section{Consent for publication}

Not required for this study.

\section{Competing interests}

The authors declare that they have no competing interests.

\section{Author details}

${ }^{1}$ The Roslin Institute, University of Edinburgh, Easter Bush campus, Midlothian, UK. ${ }^{2}$ Institute of Biological and Environmental Sciences, University of Aberdeen, Aberdeen, UK. ${ }^{3}$ Virologie et Immunologie Moleculaires, Institut National de Recherche Agronomique (INRA), Universite Paris-Saclay, Jouy-en-Josas, France. 
Received: 30 August 2019 Accepted: 10 June 2020

Published online: 23 June 2020

\section{References}

1. FAO. The state of world fisheries and aquaculture 2018-meeting the sustainable development goals: FAO; 2018. Available at: http://www.fao. org/3/19540EN/i9540en.pdf.

2. Houston RD. Future directions in breeding for disease resistance in aquaculture species. R Bras Zootec. 2017;46:545-51.

3. Yanez JM, Houston RD, Newman S. Genetics and genomics of disease resistance in salmonid species. Front Genet. 2014;5:415.

4. Zenger KR, Khatkar MS, Jones DB, Khalilisamani N, Jerry DR, Raadsma HW. Genomic selection in aquaculture: application, limitations and opportunities with special reference to marine shrimp and pearl oysters. Front Genet. 2018;9:693.

5. Knott GJ, Doudna JA. CRISPR-Cas guides the future of genetic engineering Science. 2018;361:866-9.

6. Gratacap RL, Wargelius A, Edvardsen RB, Houston RD. Potential of genome editing to improve aquaculture breeding and production. Trends Genet. 2019;35(9):672-84.

7. Datsomor AK, Zic N, Li K, Olsen RE, Jin Y, Vik JO, et al. CRISPR/Cas9-mediated ablation of elovl2 in Atlantic salmon (Salmo salar L.) inhibits elongation of polyunsaturated fatty acids and induces Srebp-1 and target genes. Sci Rep. 2019;9 https://doi.org/10.1038/s41598-019-43862-8.

8. Edvardsen RB, Leininger S, Kleppe L, Skaftnesmo KO, Wargelius A. Targeted mutagenesis in Atlantic salmon (Salmo salar L.) using the CRISPR/Cas9 system induces complete knockout individuals in the F0 generation. PLoS One. 2014;9 https://doi.org/10.1371/journal.pone.0108622.

9. Dehler CE, Lester K, Della Pelle G, Jouneau L, Houel A, Collins C, et al. Viral resistance and IFN signaling in STAT2 knockout fish cells. J Immunol. 2019; https://doi.org/10.4049/jimmunol.1801376.

10. Doench JG. Am i ready for CRISPR? A user's guide to genetic screens. Nat Rev Genet. 2018;19:67-80.

11. Fulco CP, Nasser J, Jones TR, Munson G, Bergman DT, Subramanian V, et al. Activity-by-contact model of enhancer-promoter regulation from thousands of CRISPR perturbations. Nat Genet. 2019;51(12):1664-9.

12. Orchard RC, Wilen CB, Doench JG, Baldridge MT, McCune BT, Lee YCJ, et al. Discovery of a proteinaceous cellular receptor for a norovirus. Science. 2016; 353:933-6.

13. Savidis G, McDougall WM, Meraner P, Perreira JM, Portmann JM, Trincucci G, et al. Identification of Zika virus and dengue virus dependency factors using functional genomics. Cell Rep. 2016;16:232-46.

14. Viswanatha R, Li Z, Hu Y, Perrimon N. Pooled genome-wide CRISPR screening for basal and context-specific fitness gene essentiality in Drosophila cells. Elife. 2018;7 https://doi.org/10.7554/eLife.36333.

15. Sidik SM, Huet D, Lourido S. CRISPR-Cas9-based genome-wide screening of toxoplasma gondii. Nat Protoc. 2018;13:307-23.

16. Meng $X, Y u$ H, Zhang $Y$, Zhuang F, Song X, Gao S, et al. Construction of a genome-wide mutant library in Rice using CRISPR/Cas9. Mol Plant. 2017;10: 1238-41.

17. Dehler CE, Boudinot P, Martin SAM, Collet B. Development of an efficient genome editing method by CRISPR/Cas9 in a fish cell line. Mar Biotechnol. 2016;18:449-52

18. Escobar-Aguirre S, Arancibia D, Escorza A, Bravo C, Andrés ME, Zamorano P, et al. Development of a Bicistronic vector for the expression of a CRISPR/ Cas9-mCherry system in fish cell lines. Cells. 2019;8 https://doi.org/10.3390/ cells8010075.

19. Park SW, Pyo C-W, Choi S-Y. High-efficiency lentiviral transduction of primary human $\mathrm{CD}_{3}{ }^{+}$hematopoietic cells with low-dose viral inocula. Biotechnol Lett. 2015;37:281-8.

20. Phenix KV, McKenna B, Fitzpatrick R, Vaughan L, Atkins G, Liljestrom P, et al. Cell culture evaluation of the Semliki Forest virus expression system as a novel approach for antigen delivery and expression in fish. Mar Biotechnol. 2000;2:27-37

21. Tzelepis K, Koike-Yusa H, De Braekeleer E, Li Y, Metzakopian E, Dovey OM, et al. A CRISPR dropout screen identifies genetic vulnerabilities and therapeutic targets in acute myeloid leukemia. Cell Rep. 2016;17:1193-205.

22. Sanjana NE, Shalem O, Zhang F. Improved vectors and genome-wide libraries for CRISPR screening. Nat Methods. 2014;11:783-4.

23. Yoneyama M, Kikuchi M, Natsukawa T, Shinobu N, Imaizumi T, Miyagishi M, Taira K, Akira S, Fujita T. The RNA Helicase RIG-I Has an Essential Function in
Double-Stranded RNA-Induced Innate Antiviral Responses. Nat Immunol. 2004;5(7):730-7.

24. Cronin J, Zhang $X-Y$, Reiser J. Altering the tropism of lentiviral vectors through pseudotyping. Curr Gene Ther. 2005;5:387-98.

25. Burns JC, Friedmann T, Driever W, Burrascano M, Yee JK. Vesicular stomatitis virus $G$ glycoprotein pseudotyped retroviral vectors: concentration to very high titer and efficient gene transfer into mammalian and nonmammalian cells. Proc Natl Acad Sci. 1993;90:8033-7 https://doi.org/10.1073/pnas.90.17. 8033.

26. Liu Q, Wang Y, Lin F, Zhang L, Li Y, Ge R, et al. Gene transfer and genomewide insertional mutagenesis by retroviral transduction in fish stem cells. PLoS One. 2015;10:e0127961.

27. Kawasaki T, Saito K, Mitsui K, Ikawa M, Yamashita M, Taniguchi Y, et al. Introduction of a foreign gene into zebrafish and medaka cells using adenoviral vectors. Zebrafish. 2009:6:253-8.

28. Overturf K, LaPatra S, Reynolds PN. The effectiveness of adenoviral vectors to deliver and express genes in rainbow trout, Oncorhynchus mykiss (Walbaum). J Fish Dis. 2003;26:91-101.

29. Sarmasik A, Chun CZ, Jang IK, Lu JK, Chen TT. Production of transgenic livebearing fish and crustaceans with replication-defective pantropic retroviral vectors. Mar Biotechnol. 2001;3:S177-84.

30. Naldini L, Blömer U, Gallay P, Ory D, Mulligan R, Gage FH, et al. In vivo gene delivery and stable transduction of nondividing cells by a lentiviral vector. Science. 1996;272:263-7.

31. O'Neill LS, Skinner AM, Woodward JA, Kurre P. Entry kinetics and cell-cell transmission of surface-bound retroviral vector particles. J Gene Med. 2010; 12:463-76.

32. Ott T. Tissue culture of fish cell lines. NWFHS Lab Proced Man. 2004;2:1-16.

33. O'Doherty U, Swiggard WJ, Malim MH. Human immunodeficiency virus type 1 spinoculation enhances infection through virus binding. J Virol. 2000;74: 10074-80.

34. Serebrenik Y, Sansbury SE, Santhosh Kumar S, Henao-Mejia J, Shalem O. Efficient and flexible tagging of endogenous genes by homologyindependent intron targeting. Genome Res. 2019;29(8):1322-8.

35. Pichlmair A, Diebold SS, Gschmeissner S, Takeuchi Y, Ikeda Y, Collins MK, et al. Tubulovesicular structures within vesicular stomatitis virus $G$ proteinpseudotyped lentiviral vector preparations carry DNA and stimulate antiviral responses via toll-like receptor 9. J Virol. 2007;81:539-47.

36. Schröder ARW, Shinn P, Chen H, Berry C, Ecker JR, Bushman F. HIV-1 integration in the human genome favors active genes and local hotspots. Cell. 2002;110:521-9.

37. Kim S, Kim D, Cho SW, Kim J, Kim J-S. Highly efficient RNA-guided genome editing in human cells via delivery of purified Cas9 ribonucleoproteins. Genome Res. 2014;24:1012-9.

38. Macqueen DJ, Primmer CR, Houston RD, Nowak BF, Bernatchez L, Bergseth $S$, et al. Functional annotation of all salmonid genomes (FAASG): an international initiative supporting future salmonid research, conservation and aquaculture. BMC Genomics. 2017;18:1-9.

39. Tautz D. Redundancies, development and the flow of information. Bioessays. 1992;14:263-6.

40. Barabási A-L, Oltvai ZN. Network biology: understanding the cell's functional organization. Nat Rev Genet. 2004;5:101-13.

41. Teng X, Dayhoff-Brannigan M, Cheng W-C, Gilbert CE, Sing CN, Diny NL, et al. Genome-wide consequences of deleting any single gene. Mol Cell. 2013; 52:485-94.

42. El-Brolosy MA, Kontarakis Z, Rossi A, Kuenne C, Günther S, Fukuda N, et al. Genetic compensation triggered by mutant mRNA degradation. Nature. 2019; https://doi.org/10.1038/s41586-019-1064-z.

43. Campeau E, Ruhl VE, Rodier F, Smith CL, Rahmberg BL, Fuss JO, et al. A versatile viral system for expression and depletion of proteins in mammalian cells. PLoS One. 2009;4:e6529.

44. Hsiau T, Maures T, Waite K, Yang J, Kelso R, Holden K, et al. Inference of CRISPR edits from sanger trace data. bioRxiv. 2018; https://doi.org/10.1101/ 251082.

\section{Publisher's Note}

Springer Nature remains neutral with regard to jurisdictional claims in published maps and institutional affiliations. 